Monatsschrift f. Geburtshülfe u. Gynäkologie 1924;66:I-IV

\title{
Contents, Vol. 66, 1924
}

\section{Inhaltsverzeichnis.}

Origînalarbeiten.

Seíte

Bierhoff, EUi, Die rechtliche Stellung der Hebammen . 165 Döderlein, Gustav4 siehe Voltz, Friedrich. Gänssler, Herm., Über die Schwangerschaftsreaktion von

Dienst 109

Gueissaz, Ernest, Über das Myom der Portio .... 351 Harnik, Moritz, Ovarialkarzinom neben

Karzinom der

\section{Appendix 345}

Heidler, Hans, Zur Behandlung der Nachgeburtsperiode, mit besonderer Berücksichtigung der ,Auffüllung” der Plazenta durch die Nabelschnurvene 11

Klaften, E., Untersuchungen über den respiratorischen

Gaswechsel der Schwangeren 1

Lücker, Fritz-Carl, Über Vererbung von Mißbildungen, insbesondere Hasenscharte und Polydaktylie, und ihre

Beziehungen zur Geburtshülfe 327

Mandelstamm, Alex., Zur Frage des Prolapses des Corpus luteum 223

Meier, K., Icterus neonatorum

Naujoks, Hans, Die Behandlung der Gonorrhöe der Frau. (Mit besonderer Berücksichtigung der Frisch-Vakzine-

Therapie) 31

Nissen, W., Polyzystiseher Parovialtumo,r mit eigentümlicher Wandveränderung durch Cholesterinablagerung . 339

Puppel, Ernst, Naegele-Breus-Kielland 215

Schimmel, Hugo, Eignet sich Gynergenzur Unterbrechung der Schwangerschaft?

Schoenholz, Ludwig, Zur Therapie der Placenta praevia 112 Singer, S., Ein Fall von Metastasen eines Hämangio-

endotheliom in einem Myofibrom des Uterus .... 235 Sserdjukoff, M. G., Zur Frage der

Entfernung fibrinöser,

teils ulzerierter und nekrotischer Uteruspolypen. . . 143 Stein, Arthur, Die einheitliche

Verbesserung der ameri-

kanischen Hospitaler durch das American College of

Surgeons. (Brief aus Amerika) 359

Stiglbauer, Rud., Zur Frage der Stirnhaltungsgeburt und

ihrer Behandlung 205

Strecker, Josef, Übersichtsreferat über physikalische und 
morphologische Blutfragen in der Gynäkologie ... $48>$ Unterberger, Franz, Experimentelle Zwitterbildung und

ihr Einfluß auf die Nachkommenschaft. (Ein Beitrag

zur Frage der inneren Sekretion der Keimdrüsen) . . 41 Volhard, F., Eklampsie und

Krampfurämie .... 79 Voltz, Friedr. und Gustav Döderlein, Kauterisation und

Lichtbogenoperation 247

Westphal,U., Kasuistischer Beitrag zur fötalen Peritonitis 245

IV

Inhaltsverzeichnis.

Seit $\theta$

Ko $\pi$ greß-Berichte.

Der $1 \delta$. Kongre $ß$ der deutschen Röntgengesellschaft. Berlin

27.-29. IV. $1924 \quad 363$

48. Versammlung der deutschen Gesellschaft für Chirurgie.

23.-26. IV. $1924 \quad 366$

Vereins und Literaturbeilage.

Original-Sitzungsberichte a $\mathrm{s}$ geburtshülflich-gynäkologíschen Gesellschaften:

Nordostdeutsche Gesellschaft für Gynäkologie. 55. Sitzung

vom 24. XL $1923 \quad 67$

Münchener Gynäkologische Gesellschaft. Sitzung v. 31. I.

1924175

-, Sitzung vom 13. III. 1924.307

Gynäkologische Gesellschaft in Breslau. Sitzung vom 16. X.

1923180

5

Sitzung vom 20. XI. $1923 \quad 275$

Sitzung vom 15. I. $1924 \quad 369$

Mittelrheinische Gesellschaft für Geburtshülfe und Gynäko

logie. Sitzung vom 30. VI. $1920 \quad 301$

Mitteldeutsche Gesellschaft. Sitzung in Halle am 20. I. 1924312

Gynäkologische Gesellschaft am Staatlichen klinischen

geburtshülflich-gynäkologischen Institut in Petersburg.

Sitzung vom 12. XI. $1923 \quad 316$

Der Ausschuß für $\mathrm{f}^{1} / 8$ auenwohl in New York 317

Referate aus geburtshülflich-gynäkolog. Gesellschaften . 197, 318 Geburtshülflích-

gynäkologische Mitteilungen aus anderen me-

dizinischen Gesellschaften und Vereinen . . . 198, 321, 381

Buchbesprechungen 75, 382

Liíeraturverzeichnis $76,200,322,391$

Personalien und Tagesnachrichten ... 78, 204, 326, 390, 398

Bezug von einseitig bedruckten Abzügen des Literaturberichts . 78

Druckfehler-Berichtigung $\quad 78$ 